\title{
Phase Space of Tristability in Dual Injection-Locked Fabry-Perot Laser Diodes
}

\author{
Rastko V. Pajković, Marko M. Krstić, Jasna V. Crnjanski, Angelina R. Totović, and Dejan M. Gvozdić
}

\begin{abstract}
We investigate theoretically the case of dual injection-locking, in which the two light signals are simultaneously externally injected into the cavity of a slave Fabry-Perot laser diode. We show that dual injection-locking leads to formation of new stationary points, and potentially to optical tristability of the slave laser. We show that a region in which the slave laser exhibits three stable steady-states occurs only for sufficiently different frequency detunings of the two injection signals. Moreover, the slave laser tunability depends on the choice of injection modes, and strongly depends on the slave laser bias current, with an optimal value around 2 times threshold current.
\end{abstract}

Keywords - dual injection-locking,Fabry-Perot laser diode, optical bistability, tristability.

\section{INTRODUCTION}

A LL-OPTICAL signal processing schemes, in the domain of optical communications, have been attracting more and more scientific attention in the recent years. The technique of injection-locking, in which light from one (master) laser is injected into the cavity of the slave laser, arises as a promising tool for improving various aspects of photonic components, as well as for realization of novel all-optical ones. One of the historically first noticed benefits of injection-locking is frequency chirping, linewidth, and relative intensity noise reduction in directly modulated lasers [1-3]. However, the concept of intermodal injection-locking, in which light from the master laser is injected with a frequency close to one of the slave laser's side-modes, paved the way for utilizing this technique for wavelength conversion [4], transmitter design for wavelength-division-multiplexing passive optical networks (WDM PONs) [5], Radio-over-Fiber systems [6], improved CATV transmission [7], all-optical memories [8], all-optical regenerators [9], or conversion of advanced modulation formats [10]. However, an application of particular interest in this paper is realization of an all-optical flip-flop, which is possible by applying

Paper received April 4, 2015; revised Jun 18, 2015; accepted Jun 10, 2015. Date of publication July 15, 2015. The associate editor coordinating the review of this manuscript and approving it for publication was Prof. Grozdan Petrović.

This paper is a revised and expanded version of the paper presented at the 22th Telecommunications Forum TELFOR 2014.

This work was supported by the Serbian Ministry of Education and Science (project Photonics Components and Systems 171011).

Authors are with the School of Electrical Engineering, University of Belgrade, Bulevar kralja Aleksandra 73, 11120 Belgrade, Serbia (corresponding author M. Krstić, phone: 381-11-3218444; e-mail: marko.krstic@etf.bg.ac.rs). different approaches [11], [12] which harness the optical bistability of the slave laser induced by injection-locking.

We focus on the approach that uses unidirectional intermodal, i.e., side-mode injection-locking (IL) in FabryPerot laser diode (FPLD), in which dispersive bistability provides an S-type hysteresis curve [13,14]. The model of a particular slave laser (SL) based on a multi quantum well structure[13], whose bistability has been experimentally confirmed [14],predicts a switching time shorter than 10 ps, as well as sub fJ switching energy, though not simultaneously, but at the expense of one another [15]. Further analysis indicates that the two stable states, obtained by this approach, exhibit different characteristics. A stable state close to the free-running (FR) threshold carrier concentration $n_{\text {th }}$, that is inherent to the laser even without IL, shows significant relaxation oscillations during the switching, as well as a more complicated stability map than the other stable state, which is a consequence of IL. This led us to assume that simultaneous introduction of another IL signal, to a different side-mode, could provide another stable state with more desirable characteristics. If this were so, switching between the two stable states that emerge as a consequence of dual-IL could be more time and energy efficient. Although dual-IL in FPLD has been investigated before, mainly as a mechanism that provides all-optical wavelength switching in wavelength division multiplexing [16], to the best of our knowledge, dual-IL that leads to dispersive tristability is a novel approach.

In this paper we investigate the possibility of tristability (TS) occurrence as a consequence of dual-IL in FPLD. We set the multimode rate equation (MRE) model and, through the analysis of the carrier rate $(d n / d t)$ versus the carrier concentration $(n)$, i.e., a phase plot, we find the range of injection parameters (injection power $P_{m}{ }^{\text {inj }}$ and frequency detuning $\Delta \omega_{m}$ between the master and the slave laser for both IL modes $m$, and SL bias current $I$ ) in which a single IL bistability is expanded with additional stationary points, providing the possibility for a multistable SL. By linearizing MRE system in the vicinity of steady-state solutions, under the assumption of a small perturbation, we apply the Lyapunov stability test, and find that two out of five possible steady-states are always unstable, while the remaining three steady-states can have simultaneous stability, and thus enable tristability. We analyze the influence of the injection parameters on the stability of the TS phase space. Parametric 4D tristability phase space shows that the occurrence of additional stationary states due to dual-IL is possible only when frequency detunings for side modes are sufficiently 
different. Moreover, we show that the volume of TS phase space (representing the tunabilty of SL) depends on the wavelength of the IL side modes, while the dependence on the SL bias current exhibits an optimal value for maximal tunability.

In the next section we present the model based on the multimode rate equations used for the static analysis of multistability and introduce the method for tristability range determination. We also briefly discuss the method used for stability analysis of SL stationary states. The third section deals with quantification of the SL tunability, i.e., with finding the range of operating conditions of SL for which the tristability occurs for a wider range of injection parameters. At the end, we provide conclusions of the paper.

\section{THEORETICAL MODEL}

\section{A. Multimode Rate Equation Model}

To describe the nonlinear dynamics in the slave laser that occurs upon dual- IL, a system of MREs was used similarly as in $[13,14]$. In the FR regime, this system consists of the nonlinear differential equations, which describe the rates of change of the carrier concentration $n$, photon density $S_{j}$ in each supported longitudinal mode $j$, and the phase difference $\theta_{m}$ between FR and IL case for the mode $m$ (i.e., the phase difference between FR slave and master laser), respectively. In the case of IL mode $m$, these nonlinear differential equations are extended to take into account the coupling of photon density and phase of the injected light with the already present photon density and phase of the mode of injection $m$ :

$$
\begin{aligned}
& \frac{d n}{d t}=\frac{I}{q V}-Q(n)-\sum_{j \in \mathrm{J}} v_{\mathrm{g}} g\left(n, \omega_{j}+\sum_{m \in \mathrm{M}} \delta_{j m} \Delta \omega_{m}\right) S_{j}, \\
& \frac{d S_{j}}{d t}=A_{j}(n) S_{j}+B(n)+\sum_{m \in \mathrm{M}} \delta_{j m} 2 k_{\mathrm{c}} \sqrt{S_{m} S_{m}^{\mathrm{inj}}} \cos \theta_{m}, \\
& \frac{d \theta_{m}}{d t}=\frac{\alpha A_{m}(n)}{2}-\Delta \omega_{m}-k_{\mathrm{c}} \sqrt{\frac{S_{m}^{\mathrm{inj}}}{S_{m}}} \sin \theta_{m} .
\end{aligned}
$$

In equations (1)-(3), $\delta_{j m}$ is the Kronecker delta, $S_{m}{ }^{\text {inj }}=$ $\tau_{\mathrm{p}} \Gamma P_{m}^{\mathrm{inj}} /\left(\eta_{0} \hbar \omega_{m} V\right)$ is the injected photon density in IL mode $m, g(n, \omega)$ is the material gain at the angular

\begin{tabular}{|c|c|c|c|}
\hline Symbol & Description & Value & Unit \\
\hline$\tau_{\mathrm{p}}$ & photon lifetime & 2.047 & ps \\
\hline$\Gamma$ & confinement factor & 0.056 & \\
\hline$\beta_{\mathrm{sp}}$ & $\begin{array}{l}\text { spontaneous emission coupling } \\
\text { coefficient }\end{array}$ & $2.15 \cdot 10^{-4}$ & \\
\hline$k_{\mathrm{c}}$ & external light coupling factor & $1.13 \cdot 10^{11}$ & $\mathrm{~s}^{-1}$ \\
\hline$n_{\mathrm{g}}$ & group velocity refractive index & 4.2 & \\
\hline$g_{\text {th }}$ & threshold gain & $1.22241 \cdot 10^{3}$ & $\mathrm{~cm}^{-1}$ \\
\hline$n_{\text {th }}$ & threshold carrier concentration & $2.85 \cdot 10^{18}$ & $\mathrm{~cm}^{-3}$ \\
\hline$I_{\text {th }}$ & threshold bias current & 8.2 & $\mathrm{~mA}$ \\
\hline$V$ & active area volume & $2.61 \cdot 10^{-11}$ & $\mathrm{~cm}^{3}$ \\
\hline$\alpha$ & linewidth enhancement factor & 3 & \\
\hline$A_{\mathrm{SRH}}$ & $\begin{array}{l}\text { Shockley-Reed-Hall } \\
\text { recombination constant }\end{array}$ & $1.1 \cdot 10^{8}$ & $\mathrm{~s}^{-1}$ \\
\hline$C_{\mathrm{A}}$ & Auger recombination constant & $5.82 \cdot 10^{-29}$ & $\mathrm{~cm}^{6} / \mathrm{s}$ \\
\hline$\eta_{0}$ & optical efficiency & 0.33 & \\
\hline
\end{tabular}
frequency $\omega, Q=A_{\mathrm{SRH}} n+R_{\mathrm{sp}}(n)+C_{\mathrm{A}} n^{3}$ is the total TABLE I: PARAMETER DESCRIPTION AND VALUES.

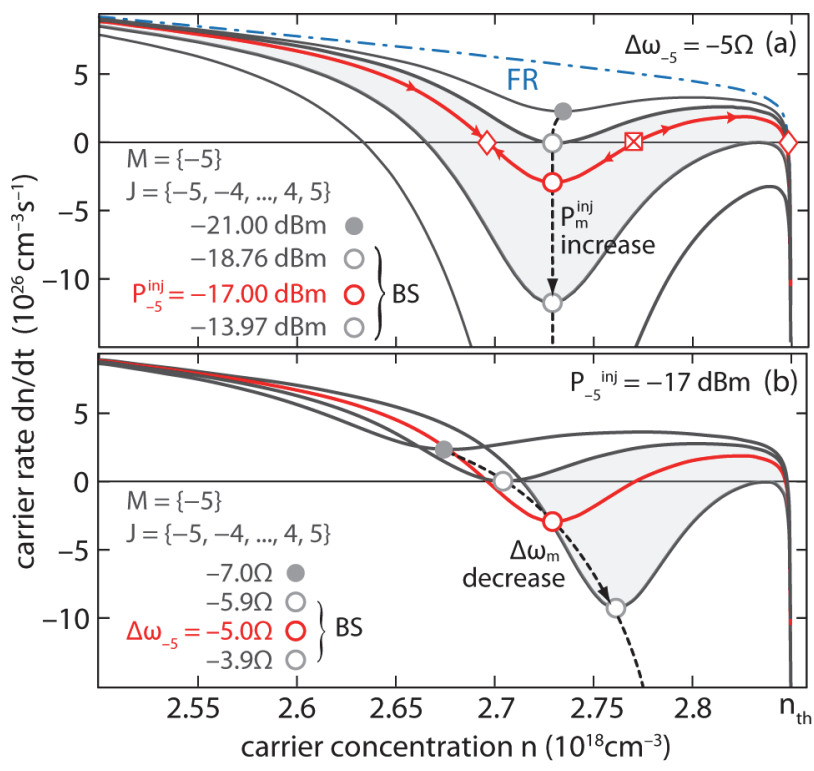

Fig. 1. Phase plots (solid lines)and trajectories of the phase plot valley minima (dashed lines) in the case of single IL into mode $\mathrm{M}=\{-5\}$, with respect to (a)injection power $P_{m}{ }^{\text {inj }}$, and (b)frequency detuning $\Delta \omega_{m}$. Phase plot in the FR regime is denoted with dash-dot line in (a).

recombination rate, $B=\Gamma \beta_{\mathrm{sp}} R_{\mathrm{sp}}(n)$ is the effective spontaneous emission, $R_{\mathrm{sp}}(n)$ represents the spontaneous recombination rate, and $A_{j}$ is the effective rate of stimulated photon generation:

$A_{j}=\Gamma v_{\mathrm{g}} g\left(n, \omega_{j}+\sum_{m \in \mathrm{M}} \delta_{j m} \Delta \omega_{m}\right)-1 / \tau_{\mathrm{p}}$.

Other parameters and their values are given in Table I.

Previous analysis [14] shows that the side modes in the vicinity of the central mode[mode with the highest gain $g(n, \omega)$ at $\left.n=n_{\text {th }}\right]$ are significantly more important for model precision than the side modes that are further apart. Therefore, in order to expedite the calculation, instead of considering all supported modes for which $g\left(n_{\mathrm{th}}, \omega\right)>0$, we choose a subset $\mathrm{J}$ that we include in the MRE system. Since we want to consider injection, with $\mathrm{M}$ we denote a subset of $\mathrm{J}$ that consists of the IL modes. Note that the number of elements in $\mathrm{M}$ ranges from 0 , in the FR case, to 2 , in the case of dual-IL.

In order to calculate $d n / d t$ versus $n$ we need to calculate $S_{j}(n)$ for every mode in J. We do this by solving the stationary form of (2), for every mode that is not in M as $S_{j}(n)=-B(n) / A_{j}(n)$. To calculate the $S_{m}(n)$ for ILmodes that are in $\mathrm{M}$, we need to solve a quadratic equation obtained by eliminating $\theta_{m}$ from the stationary forms of (2) and (3):

$\left[A_{m}^{2}+4\left(\frac{\alpha A_{m}}{2}-\Delta \omega\right)^{2}\right] S_{m}^{2}+\left(2 A_{m} B-4 k_{c}^{2} S_{\text {inj }}\right) S_{m}+B^{2}=0$,

As in [14], we choose the higher value $S_{m}=S_{m}{ }^{+}$, since $S_{m}{ }^{-}$ is in the order of magnitude of spontaneous emission. Now that we have calculated all the $S_{j}$ (including $S_{m}$ ), by using (1) we obtain a phase plot $d n / d t$ versus $n$, which provides an insight into stability of the SL.

\section{B. Phase plot analysis}

Before we analyze the case of dual IL, and discuss under which operating conditions tristability can occur, it 
is important to understand how IL affects the phase plot (Fig. 1). In the FR case[dash-dot line in Fig. 1(a)], phase plot exhibits only one stationary point, $n_{\mathrm{st}}^{(3)}=n_{\text {th }}$. Introduction of IL in the mode $\mathrm{M}=\{-5\}$ at longwavelength side with respect to the central mode, creates a valley in the phase plot(solid lines), that can lead to the occurrence of two new stationary points $n_{\mathrm{st}}{ }^{(1)} \mathrm{and} n_{\mathrm{st}}{ }^{(2)}$, as well as to a reduced value of $n_{\mathrm{st}}{ }^{(3)}<n_{\mathrm{th}}$ [14]. Analysis shows that $n_{\mathrm{st}}{ }^{(2)}$ is different from the other two stationary points in terms of stability [3].

Stability of a stationary point can be, to some extent, discussed by investigating the phase plot slope at that point. Suppose that system is settled in $n_{\text {st }}{ }^{(2)}$ and exhibits a small perturbation that leads to a slight decrease in concentration. By reading the phase plot, we can see that the rate of concentration change $d n / d t$ would become negative, causing a further decrease in concentration that would push the system away from $n_{\mathrm{st}}{ }^{(2)}$ and into $n_{\mathrm{st}}{ }^{(1)}$ (depicted with arrows on the phase plot for $P_{-5}{ }^{\text {inj }}=-17$ $\mathrm{dBm})$. By analogy, a perturbation that would increase the concentration, would push the system into $n_{\mathrm{st}}{ }^{(3)}$. Hence $n_{\mathrm{st}}{ }^{(2)}$ is a repulsive stationary point, unlike $n_{\mathrm{st}}^{(1)}$ and $n_{\mathrm{st}}^{(3)}$, that act as attractive stationary points and can be stable in terms of the small signal analysis. Detailed stability examination, based on the eigenvalue analysis [13, 14], shows that there is a region of injection power and frequency detuning in which $n_{\mathrm{st}}^{(1)}$ and $n_{\mathrm{st}}{ }^{(3)}$ are both stable, hence these parameters provide bistability (BS).

By following the valley minima, Fig. 1(a) shows how a change in $P_{-5}{ }^{\text {inj }}$ affects the phase plot. The region of interest, where BS occurs, is shaded gray and it corresponds to $P_{-5}{ }^{\text {inj }}=(-18.76,-13.97) \mathrm{dBm}$ range. In this BS region, a change in $P_{-5}{ }^{\text {inj }}$ results in a change of the valley depth, without changing the valley minima position with respect to $n$.The same is not true for a change in detuning $\Delta \omega_{-5}$ as Fig. 1(b) shows. The minus sign in the detuning value means that the frequency of injected light is lower than the frequency of the mode of injection. Note that detuning of $\Delta \omega_{-5}=-5 \Omega\left(\Omega=10^{10} \mathrm{rad} / \mathrm{s}\right)$ is lower than $\Delta \omega_{-5}=-7 \Omega$ since it denotes an injection frequency closer to the frequency of the mode of injection. This means that, in the BS region, phase plot valley becomes shallower with higher detuning $\Delta \omega_{m}[$ c.f. Fig. 1(b)], and in order to deepen it back, it is necessary to increase the injection power $P_{m}{ }^{\text {inj }}$ [c.f. Fig. 1(a)]. Analysis of the entire range of injection parameters shows that this conclusion is true in general [14]. The third parameter of injection, IL modem, does not have a significant impact on the phase plot, in the range of -10 to 10 . It tends to shift the entire valley slightly towards the higher $n$, without altering its shape significantly. In summary, there are two important conclusions that come from analyzing Fig. 1: $(i)$ we can shift the valley towards the higher $n$ with a lower (less negative) $\Delta \omega_{m}$, and (ii) in the region with several stationary points, a higher (more negative) $\Delta \omega_{m}$ requires a higher $P_{m}^{\text {inj }}$ in order to achieve BS.

Based on the previous analysis of single side-mode IL, it is expected that injection of the second optical signal will create another valley in the phase plot, providing two additional stationary points, i.e., five in total. Fig. 2 shows how injection in a second mode creates $n_{\mathrm{st}}{ }^{(3)}$ and $n_{\mathrm{st}}{ }^{(4)}$, out of which only $n_{\mathrm{st}}{ }^{(3)}$ is stable, in addition to the other two stable stationary points $n_{\mathrm{st}}^{(1)}$ and $n_{\mathrm{st}}{ }^{(5)}-$ hence tristability.

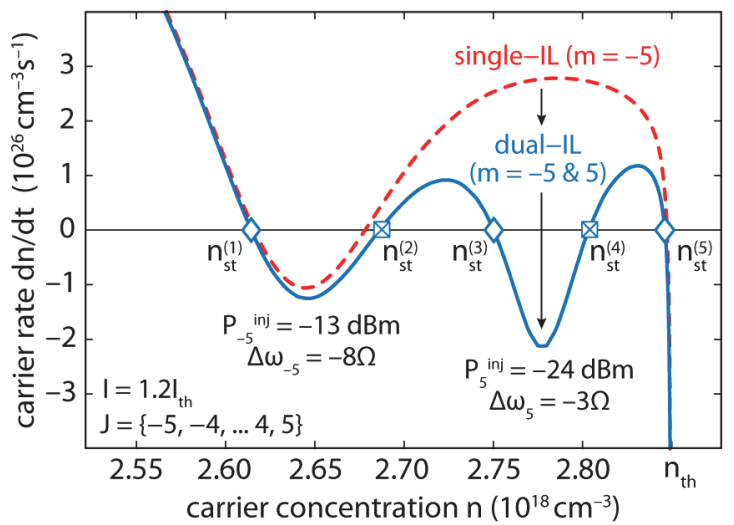

Fig. 2. $d n / d t$ phase plot in the case of single IL (dashed line) and with dual-ILin modes $\mathrm{M}=\{-5,5\}$ (solid line).

Since $n_{\mathrm{st}}{ }^{(5)}$ (similar to $n_{\mathrm{st}}{ }^{(3)}$ in the case of single IL) is always close to $n_{\text {th }}$ we can say that it is inherent to laser in the FR case, while $n_{\mathrm{st}}{ }^{(1)}$ and $n_{\mathrm{st}}{ }^{(3)}$ are consequences of IL. Based on $(i)$,one can deduce that the valley on the right (higher $n$ ) comes from injection in mode $m=5$, because this injected signal has a lower $\Delta \omega_{m}$. Conclusion $(i i)$ also holds true, as the injected signal with a higher $\Delta \omega_{m}(m=$ -5) has a higher $P_{m}$ inj.

Having proved that dual-IL can provide extra stationary points, and thus provide TS, our goal is to determine the range of injection parameters for which this occurs. Due to another injected signal in the case of dual-IL, in order to determine the TS range 7 injection parameters need to be simultaneously varied. These parameters are: SL bias current $I$, and 3 parameters for each injected signal - mode of injection $m$, detuning $\Delta \omega_{m}$, and injected $\operatorname{power} P_{m}$ inj. Bias current from $I=1.2 I_{\text {th }}$ to $I=5 I_{\text {th }}$ is considered. Injection is performed in modes ranging from $m=-10$ to $m=10 . P^{\text {inj }}$ is varied from $-30 \mathrm{dBm}$ to $15 \mathrm{dBm}$, where the upper limit is chosen so that the nonlinear gain suppression is not triggered. Considering that intermodal space of the SL is $89.7 \Omega$, and that the positive detuning is of no interest $[13,14]$, we considered detuning from $0 \Omega$ to $-44 \Omega(\approx 89.7 \Omega / 2)$. For each set of parameters we then calculate the number of stationary points that occur in phase plot .Since $\Delta \omega_{m}$ and $P_{m}$ inj are of particular interest, we chose a denser discretization of 40 equidistant points for these parameters.

\section{Stability analysis}

On the basis of the phase slope investigation it can be concluded that in the case of dual injection-locked FabryPerot laser diodes, $n_{\mathrm{st}}{ }^{(2)}$ and $n_{\mathrm{st}}{ }^{(4)}$ points are repulsive stationary points, while the remaining three steady-states act as attractive stationary points. However, in order to investigate the stability in the sense of Lyapunov, we apply the Lyapunov stability test. We assume a small perturbation of the system, i.e., we assume that injection modes photon density $\left(S_{m}\right)$, unlocked modes photon density $\left(S_{j}\right)$, carrier concentration $(n)$, injection modes phase $\left(\theta_{m}\right)$, and slave laser bias current $(I)$ exhibit a small variation $\left(\delta S_{m}, \delta S_{j}, \delta n, \delta \theta_{m}\right.$ and $\delta I$, respectively) around their steady-state values $\left(S_{m}{ }^{(p)}, S_{j}^{(p)}, n_{\mathrm{st}}{ }^{(p)}, \theta_{m}{ }^{(p)}\right.$, and $I_{\mathrm{dc}}$, respectively), where $p=1,2,3,4$ or 5. Time evolutions of these quantities can be expressed as $\chi(t)=X^{(p)}+$ $\delta \chi^{(p)} \exp (\xi t)$, where $X^{(p)}$ stands for the steady-state of the particular quantity, $\delta \chi^{(p)}$ stands for the amplitude of the 
corresponding small variation, while $\xi$ represents the eigenvalue of the system. We linearize the rate equation system around the $\chi(t)$ values, which yields a system of linear homogenous equations with respect to small variations $\delta \chi$ and complex eigenvalues $\xi$, from which we solve for $\xi$.

A steady-state is considered to be dynamically stable if all eigenvalues lie in the left-half of the $\xi$-complex plane, i.e., if all real parts of complex eigenvalues have negative values.

\section{TS OCCURRENCE RANGE AND ITS QUANTIFICATION}

Our main interest and the key result of this paper is the range of parameters for which TS occurs. The goal is to recognize conditions for obtaining larger SL "tunability", meaning that TS occurs for a wider range of injection parameters. Moreover, of particular interest is to investigate to what extent instabilities impact the TS phase space, and thus reduce the SL tunability.

Instead of finding a way to visualize 7D data, which proves to be ineffective, we quantify the volume of $4 \mathrm{D}$ phase space (defined with $\Delta \omega_{m}$ and $P_{m}{ }^{\text {inj }}$ for each of the two IL modes $m$ ) with respect to the remaining 3 parameters. This allows us to see how SL bias current and the choice of IL side modes affect the range of TS tunability. Since points in the range of $\Delta \omega_{m}$ and $P_{m}$ inj are equidistant for both injected modes, we calculate the volume of one point in 4D phase space as a product of step between the two nearest points in every dimension, or $V_{\text {point }}=\Delta P_{m 1}{ }^{\text {inj }} \times$ $\Delta P_{m 2}{ }^{\text {inj }} \times \Delta\left(\Delta \omega_{m 1}\right) \times \Delta\left(\Delta \omega_{m 2}\right)$. The entire volume of TS that a set of 3 remaining parameters define is then calculated as $V=$ (number of points in $4 \mathrm{D}$ phase space) $\times V_{\text {point }}$. This allows us to compare different volumes and thus quantify the tunability that the chosen SL bias current and IL sidemodes provide.

Fig. 3(a) shows an example of 4D phase space of TS for injection into $\mathrm{M}=\{-5,5\}$, at $I=3 I_{\text {th }}$. Corresponding side views of 4D phase space are given in Figs. 3(b) and (c). The difference between the values of the frequency detunings in IL side-modes is a limiting factor for theTS occurrence, since TS cannot be obtained when the frequency detuning for both injection signals is equal, or close to equal, i.e. $\Delta \omega_{m 1} \approx \Delta \omega_{m 2}$ [c.f. Fig. 3(b)].The reason for this lies in the way that valley of the $d n / d t$ versus $n$ phase plot shifts with a change in $\Delta \omega_{m}$, as explained in Fig. 1. In order to add extra stationary points in the phase plot, two valleys have to be sufficiently separated so they cannot merge into one valley, thus providing two, instead of four stationary points. Furthermore, it can be seen that the greater detuning corresponds to the greater "forbidden TS area" where TS does not occur.

In addition to this, we analyze where in the $P_{m}{ }^{\text {inj }}$ versus $\Delta \omega_{m}$ space (corresponding to the one of the injected signals) TS occurs [c.f. Fig. 3(c)]. This space has been closely inspected in case of the single injection [14], and it was shown to provide BS in an area that resembles a tip of a curved sword, with ever-expanding width. Fig. 3(c) shows that dual injection resembles this familiar shape, now affected also by the second injection power.

In order to quantify and measure the tunability of the slave laser under the dual-IL, we present normalized volumes for different values of SL bias current $I$, and different pairs of IL side-modes (Fig. 4). Volumes are normalized with respect to the maximal calculated volume $\left(V_{\max }\right)$ which is found for injection into $\mathrm{M}=\{0,5\}$, at $I=$ $3 I_{\text {th }}$. The plot shows that an increase in $I$, provides an increase in tunability, i.e., a greater volume, but only up to $I=3 I_{\text {th }}$, where the system shows the largest tunability, regardless of the chosen injection modes. The explanation for these local maxima lies in the fact that larger bias currents require stronger injection powers, which imply more negative values of $\Delta \omega_{m}$ in order to achieve TS. However, for sufficiently large bias currents, this negative value of $\Delta \omega_{m}$ exceeds the boundaries of the intermodal space, hence in this case we assume that there is no occurrence of multistablity, which leads to the volume shrinkage for currents above $3 I_{\text {th }}$. Closer inspection of Fig. 4 shows that pairs of positive modes of injection $\mathrm{M}=\{0$, $5\}$ and $\{5,10\}$ provide more tunability (greater volume) than the corresponding pairs of negative modes $\mathrm{M}=\{0,-$ $5\}$ and $\{-5,-10\}$. Further the modes are from the central mode, as is the case for $\mathrm{M}=\{5,10\}$, less tunability they provide, which can be seen in Fig. 4, by comparing with $M$ $=\{0,5\}$. Greater tunability for positive modes (shortwavelength) is an effect inferred from the single injectionlocking [14], where it can be seen that the region of bistability is larger in the case of positive longitudinal modes, and reduces as modes are shifted away from the central one. Larger tunability of the positive modes in comparison to equivalent negative ones is a consequence of the asymmetric gain profile in favor of positive modes, which are more easily triggered by the injection due to a smaller gain defect. A smaller gain defect is also responsible for lager tunability of the modes in the vicinity of the central longitudinal mode.

In this range of TS we conduct the Lyapunov stability analysis. The stability analysis confirms conclusions
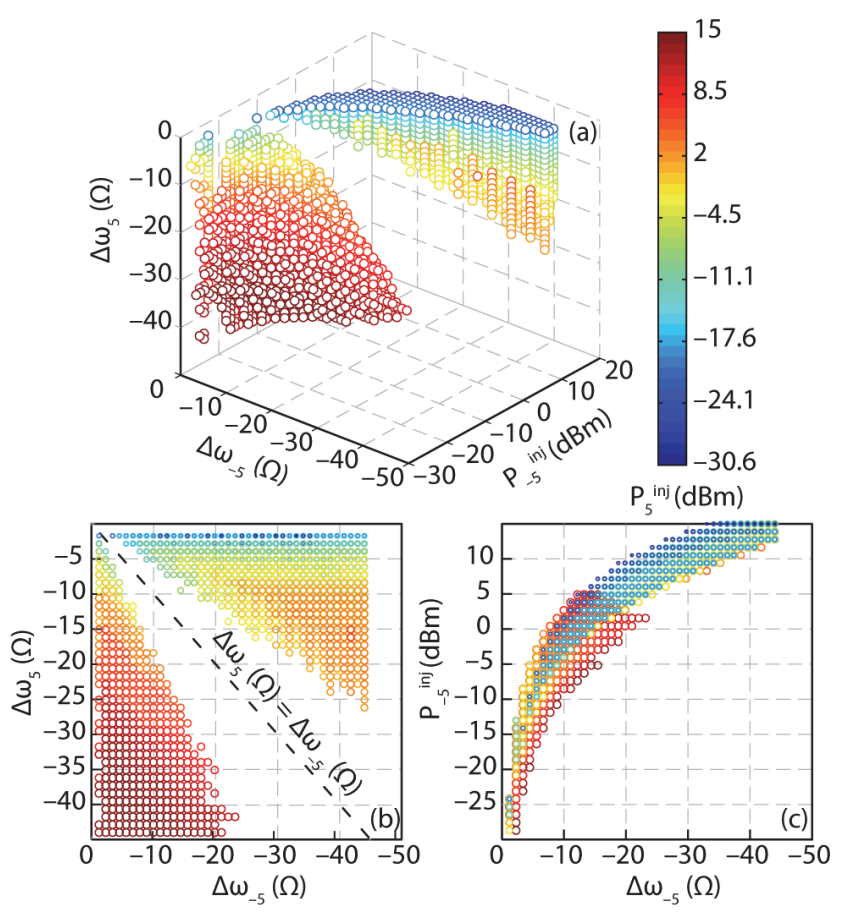

Fig. 3.(a) $4 \mathrm{D}$ phase space of $\mathrm{TS}$ for injection into $\mathrm{M}=$ $\{-5,5\}$ at $I=3 I_{\text {th. }}$. Side-views of $4 \mathrm{D}$ phase space: (b) frequency detuning $\Delta \omega_{5}$, and (c) injection power $P_{-5}{ }^{\text {inj }}$, with respect to $\Delta \omega_{-5}$ and $P_{5}{ }^{\text {inj }}$. The fourth dimension (injection power $P_{5}{ }^{i n j}$ ) is encoded with color. 
deduced from the phase plot slope, and shows that $n_{\mathrm{st}}{ }^{(2)}$ and $n_{\mathrm{st}}^{(4)}$ are always unstable. On the other hand, $n_{\mathrm{st}}^{(1)}$ and $n_{\mathrm{st}}{ }^{(3)}$ are always stable, thus always providing bistability of dual injection-locked Fabry-Perot laser diodes. Depending on the injection parameters, $n_{\mathrm{st}}^{(5)}$ point can be dynamically stable and provide the Lyapunov tristability of the system.

The stability analysis reveals that the stability of the $n_{\mathrm{st}}^{(5)}$ point is mainly influenced by the injection power and frequency detuning responsible for creating the "valley" in the vicinity of the $n_{\mathrm{st}}^{(5)}$ point. According to the conclusion (i) from the section II.B., the responsible set of parameters are the smaller frequency detuning and corresponding injection power (c.f. Fig. 2). Due to the fact that in the region of TS, the two frequency detunings have significantly different values [c.f. Fig. 3(b)], the second "valley" is separated from the $n_{\mathrm{st}}^{(5)}$ "valley" enough so that the variation of the larger frequency detuning, and corresponding injection power, has almost no influence on the $n_{\mathrm{st}}^{(5)}$ point, and thus its stability. In that manner, the stability behavior of the $n_{\mathrm{st}}^{(5)}$ point is very similar to the behavior in the case of single injection-locking [13], meaning that the point is unstable for small negative frequency detuning, while it exhibits stability, and provides the Lyapunov tristability for sufficiently large negative frequency detuning.

From the practical point of view, it is of importance to investigate the magnitude of stability occurrence in the region of TS. Fig 5. shows the ratio of Lyapunov stable volume $V_{\mathrm{LSTS}}$ to tristability volume $V_{\mathrm{TS}}$, expressed in percent, with respect to normalized SL bias current, for injection into different modes. It can be seen that injection which includes the central mode $(\mathrm{M}=\{0,5\}$ and $\{0,-5\})$ has the lowest percent of stability, while the symmetrical injection-locking $(\mathrm{M}=\{5,-5\})$ has the highest percent of stability. Injection into short-wavelength side modes $(\mathrm{M}=$ $\{5,10\})$ has a slightly higher percent of stability compared to injection into longer-wavelength modes $(\mathrm{M}=\{-5$, $10\})$. Stability generally steeply decreases with an increase in the SL bias current, and for $I=5 I_{\mathrm{th}}$, below $1 \%$ of the TS volume is stable.

Fig. 6. shows the normalized volume in which all three attracting steady-state points $\left(n_{\mathrm{st}}^{(1)}, n_{\mathrm{st}}^{(3)}\right.$ and $\left.n_{\mathrm{st}}^{(5)}\right)$ exhibit stable dynamics. In order to compare Fig. 6 with Fig. 4. the calculated Lyapunov stable volumes $V_{\text {LSTS }}$ in Fig. 6. are normalized to the same maximal calculated volume $V_{\max }$. Since there exists a high discrepancy between the percent of the stability obtained for injection including the central mode, and symmetrical injection, as opposed to the results depicted in Fig. 4., symmetrical injection emerges as the one which provides the greatest tunability of the SL (c.f. Fig. 6). In addition to this, the discrepancy in the terms of SL tunability between injection into $\{0,-5\}$ and $\{5,10\}$ is highly reduced. Finally, the optimal value of SL bias current is shifted towards smaller values, from $3 I_{\text {th }}$ to about $2 I_{\text {th }}$, which gives around $60 \%$ of stable TS volume. High bias currents significantly reduce the stable portion of the TS volume, leading to below $1 \%$ stability for $I=$ $5 I_{\text {th }}$.

\section{CONCLUSION}

Dual injection-locking imposes itself as an efficient mechanism for achieving optical tristability. In this paper we show that for a certain range of injection parameters, as

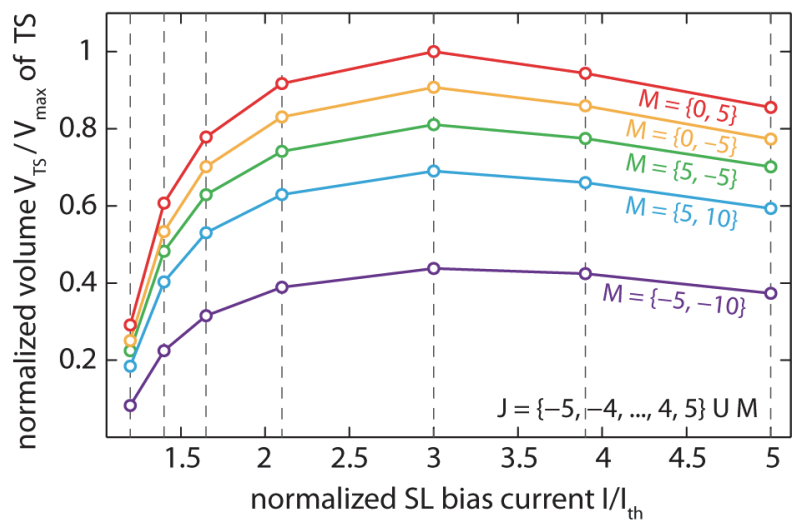

Fig. 4. Normalized volume $V_{\mathrm{TS}} / V_{\max }$ as a measure of tunability, versus normalized SL bias current $I / I_{\text {th }}$ for different IL modes M.

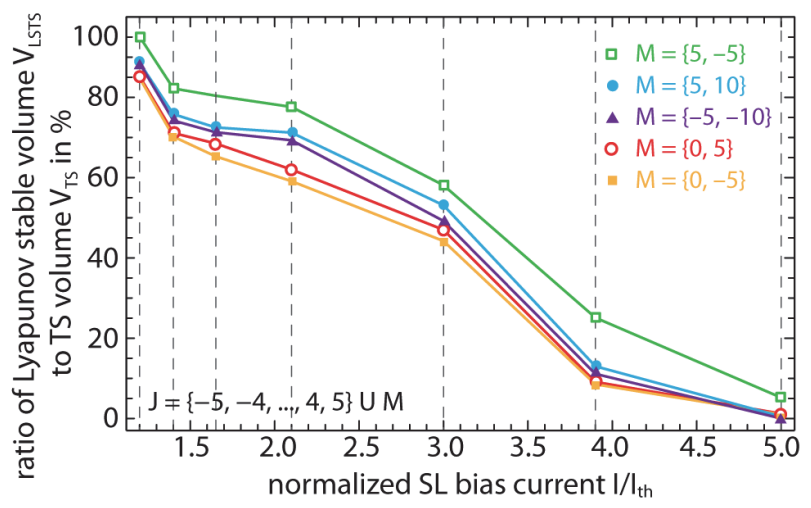

Fig. 5. Ratio of Lyapunov stable volume ( $\left.V_{\text {LSTS }}\right)$ to TS volume $\left(V_{\mathrm{TS}}\right)$ versus normalized SL bias current $I / I_{\mathrm{th}}$ for different IL modes M.

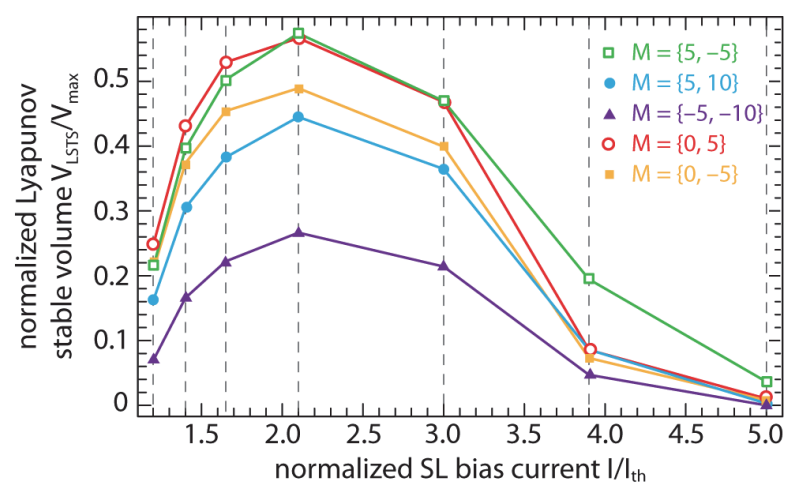

Fig. 6. Normalized Lyapunov stable volume $V_{\text {LSTS }} / V_{\max }$ as a measure of tunability versus normalized SL bias current $I / I_{\text {th }}$ for different IL modes $\mathrm{M}$.

a consequence of dual injection-locking, a slave laser can have up to 5 stationary points, 3 of which can be stable. As in the case of single injection-locking, where we perform switching between two stable states [15], switching between these three states can also be performed by variation of injection power or frequency detuning.

The main focus of the paper is an investigation of the phase space in which tristability exists. We analyze the influence of injection parameters on the phase space in order to achieve maximal slave laser flexibility, i.e. "tunability" in terms of tristability occurrence and dynamical stability, since the region in which this third 
state exhibits stable dynamics is of high importance for advanced optical and logical functions in advanced optoelectronic integrated devices used in optical signal processing [17]. We show that values of the frequency detunings limit the possibility of achieving slave laser tristability, i.e. tristability cannot be achieved for close values of the two frequency detunings. Furthermore, we show that the bias current impacts the volume of the parametric space for which the tristability exists, making this volume the largest for $3 I_{\text {th }}$, and thus providing the largest tunability. In addition to this, positive (shortwavelength) longitudinal modes provide larger tunability in comparisonwith the negative longitudinal modes (longwavelength). The tunability becomes larger as injection mode becomes closer to the central longitudinal mode.

Stability analysis shows that in the range of optical tristability two points always exhibit stable dynamics, and that the third point is stable for sufficiently large negative detuning. High slave laser bias currents significantly pronounce instabilities, leading to the almost complete cancelation of the tristability for the currents higher than $5 I_{\text {th }}$. The largest volume of Lyapunov stability in the tristability domain is obtained for the current of about $2 I_{\text {th }}$. In addition to this, injection-locking, which comprises modes on the both sides of the central mode, provides the highest percent of stability, while injection-locking, which comprises the central mode, provides the lowest percent of stability.

\section{REFERENCES}

[1] C. Lin, F. Mengel, "Reduction of frequency chirping and dynamic linewidth in high-speed directly modulated semiconductor lasers by injection locking," Electron. Lett., vol. 20, pp. 1073-1074, 1984.

[2] S. Piazzolla, P. Spano, M. Tamburrini, "Small signal analysis of frequency chirping in injection-locked semiconductor lasers," IEEE J. Quantum Electron., vol. QE-22, pp. 2219-2223, 1986.

[3] X. Jin, S. L. Chuang, "Relative intensity noise characteristics of injection-locked semiconductor lasers," Appl. Phys. Lett., vol. 77, pp. 1250-1252, 2000.
[4] C. W. Chow, C. S. Wong, H. K. Tsang, "All-optical data-format and wavelength conversion in two-wavelength injection-locked slave Fabry-Perot laser diodes," Electron. Lett., vol. 39, pp. $997-$ 999, 2003.

[5] N. Kashima, "Dynamics properties of FP-LD transmitters using side-mode injection locking for LANs and WDM-PONs," $J$. Lightw. Technol., vol. 24, pp. 3045-3058, 2006.

[6] A. Kaszubowska, P. Anandarajah, L. P. Barry, "Improved performance of a hybrid radio/fiber system using a directly modulated transmitter with external injection," IEEE Photon. Technol. Lett.,vol. 14, pp. 233-235, 2002.

[7] L. Hai-Han, H. Hsu-Hung, S. Heng-Sheng, W. Ming-Chuan, "Fiber optical CATV system-performance improvement by using external light-injection technique," IEEE Photon. Technol. Lett., vol. 15, pp. 1017-1019, 2003.

[8] S. Osborne, K. Buckley, A. Amann, S. O'Brien, "All-optical memory based on the injection locking bistability of a two-color laser diode," Opt. Exp., vol. 17, pp. 6293-6300, 2009.

[9] K. Huybrechts, T. Tanemura, K. Takeda, Y. Nakano, R. Baets, G. Morthier, "All-optical 2R regeneration using the hysteresis in a distributed feedback laser diode," IEEE J. Sel. Topics Quantum Electron., vol. 16, pp. 1434-1440, 2010.

[10] C. W. Chow, C. S. Wong, H. K. Tsang, “All-optical modulation format conversion and multicasting using injection-locked laser diodes," J. Lightw. Technol., vol. 22, pp. 2386-2392, 2004.

[11] Y.D. Jeong, J.S. Cho, Y.H. Won, H.J. Lee, H. Yoo, "All-optical flip-flop based on the bistability of injection locked Fabry-Perot laser diode," Opt.Exp., vol. 14.9, pp. 4058-4063, 2006.

[12] L. Liu, R. Kumar, K. Huybrechts, T. Spuesens, G. Roelkens, E.J. Geluk et al., "An ultra-small, low-power, all-optical flip-flop memory on a silicon chip," Nature Photonics, vol. 4, pp. 182-187, 2010.

[13] M.M. Krstić, J.V. Crnjanski, M.L. Mašanović, L.A. Johansson, L.A. Coldren, D.M. Gvozdić, "Multivalued stability map of an injection-locked semiconductor laser", IEEE J.Sel.TopicsQuantum Electron., vol. 19, pp. 1501408, 2013.

[14] M.M. Krstić, J.V. Crnjanski, D.M. Gvozdić. "Injection power and detuning-dependent bistability in Fabry-Perot laser diodes," $\mathrm{J}$. Sel. Topics Quantum. Electron., vol. 18, pp. 826-833, 2012.

[15] M.M. Krstić, J.V. Crnjanski, D.M. Gvozdić,"Switching time and energy in bistable injection-locked semiconductor multi-quantumwell Fabry-Perot lasers."Phys. Rev. A, vol. 88, pp. 063826, 2013.

[16] H.K. Tsang, L.Y. Chan, S.P. Yam, C. Shu, "Experimental characterization of dual-wavelength injection-locking of a FabryPerot laser diode," Optics communications, vol. 156.4, pp. 321-326, 1998.

[17] K. Hybrechts, B. Maes, G. Morthier, R. Baets, "Tristable all-opitcal flip-flop using coupled nonlinear cavities," IEEE/LEOS Winter Topical Meeting Series, pp. 16-17, 2008. 